\title{
CAPACIDADE COMBINATÓRIA, DIVERGÊNCIA GENÉTICA ENTRE LINHAGENS DE MILHO E CORRELAÇÃO COM HETEROSE $\left({ }^{1}\right)$
}

\author{
MARIA ELISA AYRES GUIDETTI ZAGATTO PATERNIANI $\left(\left(^{*}\right)\right.$; PAULA DE SOUZA GUIMARÃES $\left({ }^{3}\right)$; \\ REGINALDO ROBERTO LÜDERS $\left({ }^{2}\right)$; PAULO BOLLER GALLO $\left({ }^{4}\right)$; ANETE PEREIRA DE SOUZA $\left({ }^{5}\right)$; \\ PRIANDA RIOS LABORDA $\left({ }^{5}\right)$; KARINE MIRANDA OLIVEIRA $\left({ }^{5}\right)$
}

\begin{abstract}
RESUMO
Objetivou-se avaliar o desempenho de híbridos simples de milho (Zea mays L.), obtidos de cruzamentos dialélicos entre linhagens divergentes, estimar a capacidade de combinação das linhagens e verificar se a divergência genética entre as linhagens, obtida por marcadores moleculares, é correlacionada com a heterose dos híbridos simples no campo. Trinta e seis híbridos resultantes de um dialelo parcial foram avaliados em Campinas e em Mococa e as 12 linhagens parentais somente em Campinas, a fim de se calcular a heterose dos híbridos. O delineamento experimental empregado foi o de blocos ao acaso, com três repetições e 2 testemunhas comerciais. Avaliaram-se os caracteres: altura da planta (AP), altura da espiga (AE) e massa de grãos (MG). Análises de variância foram efetuadas, sendo as médias comparadas entre si pelo teste de Tukey a 5\%. A capacidade de combinação das linhagens foi obtida de acordo com o método de Geraldi e Miranda Filho. Estimaram-se as correlações entre heterose, produtividade e capacidade específica de combinação com divergência genética obtida por AFLP e SSR. Destacou-se o híbrido PM624 x IP398, e as linhagens VER266 e L105 revelaram efeitos positivos da capacidade de combinação para produtividade. As estimativas de heterose variaram de -559 a $6.320 \mathrm{~kg} \mathrm{ha}^{-1}$. Não houve correlação entre heterose, capacidade específica de combinação e produtividade dos híbridos com a distância genética por AFLP e SSR, indicando que não é possível fazer inferências sobre o comportamento dos híbridos de milho a partir da divergência genética entre as linhagens parentais.
\end{abstract}

Palavras-chave: Zea mays, dialelo parcial, produtividade, AFLP, SSR.

\section{ABSTRACT \\ COMBINING ABILITY, GENETIC DIVERGENCE AMONG MAIZE LINES AND CORRELATION WITH HETEROSIS}

The objectives of this research were to evaluate single cross hybrids of maize (Zea mays L.) obtained from partial diallel crosses among contrasting inbred lines, to estimate the combining ability of the lines and to verify whether the genetic diversity among those lines assessed by moleculars markers is correlated with single cross hybrid heterosis Thirty-six single-crosses resulting from partial diallel and the 12 parental lines were evaluated in Campinas, State of São Paulo, in randomized block design, with tree replicates and two commercial checks. The following traits were evaluated: plant and ear height, ear weight and grain weight. Individual variance analysis of hybrids were performed, and the averages were compared by Tukey test $(\mathrm{P}<0.05)$. General combining ability of the lines was obtained according to Geraldi e Miranda Filho. Correlations among matrices were estimated through Mantel statistics,

$\left({ }^{1}\right)$ Recebido para publicação em 20 de junho de 2007 e aceito em 18 de março de 2008.

$\left({ }^{2}\right)$ Centro de Análise e Pesquisa Tecnológica do Agronegócio de Grãos e Fibras, Instituto Agronômico (IAC), Av. Barão de Itapura, n.1481, 13020-902, Campinas (SP). E-mail: elisa@iac.sp.gov.br $\left(^{*}\right)$ Autora correspondente; rrluders@iac.sp.gov.br.

$\left({ }^{3}\right)$ Instituto Agronômico (IAC), Pós Graduação em Agricultura Tropical e Subtropical, Campinas (SP). E-mail: psguim@yahoo.com.br.

$\left({ }^{4}\right)$ Pólo Regional de Desenvolvimento do Nordeste Paulista (APTA), Caixa Postal 58, 13730-970 Mococa (SP). E-mail: polonordestepaulista@aptaregional.sp.gov.br

$\left({ }^{5}\right)$ Centro de Biologia Molecular e Engenharia Genética, Universidade de Campinas (UNICAMP). E-mail: anete@unicamp.br; prianda@unicamp.br; kmoliveira@unicamp.br 
considering heterosis, yield and specific combining ability with genetic divergence assessed by AFLP and SSR. The hybrid PM624 x IP398 exhibited outstanding yield capacity and the lines VER266 and L105 showed positive GCA for grain yield. Estimate heterosis ranged from -559 to $6.320 \mathrm{~kg} \mathrm{ha}^{-1}$. No significant correlation was observed between heterosis, specific combination ability or grain yield with genetic distance assessed by AFLP and SSR. Therefore, no prediction of hybrids performance could be made on the basis of the genetic divergence of the parent lines.

Key words: Zea mays, partial diallel, yield, AFLP, SSR.

\section{INTRODUÇÃO}

O emprego de linhagens em programas de melhoramento de milho está associado à busca do vigor de híbrido, ou heterose, fenômeno que proporciona grande produtividade em híbridos provenientes do cruzamento de parentais que exibem alta divergência entre si.

A teoria da genética quantitativa, descrita por FALCONER (1981) sugere que, em qualquer grau de dominância superior a zero, a heterose é uma função da freqüência alélica entre os genitores, existindo, portanto, uma correlação positiva entre divergência genética e heterose. Por esse motivo, estimativas de diversidade entre linhagens vêm sendo utilizadas como meio de predição de cruzamentos superiores em programas de melhoramento de plantas (Hallauer e Miranda Filho, 1995).

A avaliação das linhagens quanto à capacidade de combinação é a etapa mais trabalhosa e onerosa dos programas de híbridos na cultura do milho. O método de cruzamentos dialélicos proposto por GRIFFING (1956 a, b) baseia-se nos conceitos de capacidade geral e específica de combinação estabelecidos por SPRAGUE e TATUM (1942). Estes autores definiram capacidade geral de combinação (CGC) como o comportamento médio de uma linhagem em combinações híbridas e capacidade específica (CEC) como o comportamento que leva certas combinações híbridas a serem superiores ou inferiores em relação à média das linhagens envolvidas.

O método de cruzamentos dialélicos permite ainda estimar os efeitos da CGC, que proporcionam informações sobre a concentração de alelos predominantemente aditivos, e os efeitos da CEC, que evidenciam a importância dos efeitos não-aditivos ou predominantemente em dominância ou epistasia (CRUZ e RegazZI, 1997).

Na prática, os dialélicos completos limitam o número de linhagens a serem utilizadas, requerendo muito esforço nas polinizações manuais para obtenção de todos os cruzamentos desejados. Nos programas de obtenção de híbridos, é comum se realizarem cruzamentos entre germoplasmas divergentes, visando à maximização da heterose.
Assim, os cruzamentos dialélicos intrapopulacionais foram substituídos por novos esquemas de dialélicos parciais, com utilização de populações diferentes para obtenção de híbridos.

VENCOVSKY (1970) sugeriu a avaliação da capacidade geral e específica de combinação de dois conjuntos de variedades, em que são avaliados somente os híbridos intervarietais e estendeu o termo "cruzamentos dialélicos" para o intercruzamento de linhagens com qualquer grau de endogamia ou mesmo para variedades. Miranda Filho e Geraldi (1984) propuseram um modelo para análise de dialélicos parciais entre grupos distintos de variedades, como adaptação da análise do dialélico completo de GARDNER E EBERHART (1966). Uma adaptação do método 2 do modelo de GRIFFING (1956b), onde se avaliam as médias das variedades e dos híbridos intergrupos, foi apresentada por Geraldi e Miranda Filho (1988).

Mais recentemente, ainda tentando facilitar a etapa de avaliação e escolha de linhagens quanto à capacidade de combinação para maximizar a heterose dos híbridos, têm sido muito utilizados pelos melhoristas métodos de cruzamentos dialélicos associados aos marcadores moleculares, com a finalidade de avaliar a divergência genética entre as linhagens endogâmicas, bem como separar essas linhagens em grupos heteróticos distintos (BARBOSA et al., 2003).

Estudos realizados com linhagens obtidas das populações BR-105 e BR-106, correlacionando produtividade e divergência genética estimada através de marcadores moleculares do tipo RFLP, RAPD, microssatélites e AFLP (LANZA et al., 1997; BENCHIMOL et al., 2000) indicaram que os melhores marcadores para alocar as linhagens em grupos heteróticos são os microssatélites e AFLP, sendo ambos complementares.

Existem trabalhos relatando que quanto maior a divergência genética entre os parentais, maior será a heterose e, conseqüentemente, a produção do híbrido (Maluf et al., 1983; Oliveira, 1989). Entretanto, nem sempre a correlação entre divergência genética obtida por marcadores moleculares e desempenho do híbrido é consistente (Barbosa et al., 2003, Melo et al., 2001, GuimarÃes, 2007). 
De acordo com GADHERI et al. (1984), é necessário haver efeitos de dominância ou de epistasia envolvendo dominância para que ocorra uma associação positiva entre divergência genética e a heterose, e que os parentais sejam diferentes nas freqüências alélicas que controlam o caráter em estudo. Essa diferença deve aumentar com a divergência dos parentais, permitindo com que os efeitos de dominância se manifestem e, consequentemente, o fenômeno da heterose seja aumentado.

Melo et al. (2001) não observaram correlação entre distância de Malahanobis entre híbridos comerciais de milho com efeitos de capacidade específica de combinação ou com heterose. GUIMARÃES et al. (2007) obtiveram correlação da divergência genética entre linhagens de milho obtida por AFLP e SSR apenas com heterose, mas verificaram que altas estimativas de distância genética não se correlacionaram com capacidade específica de combinação das linhagens nem com a produtividade dos híbridos simples.

Assim, o presente trabalho tem por objetivo avaliar híbridos simples obtidos de cruzamentos dialélicos entre linhagens divergentes, sendo esta divergência estimada a partir de marcadores moleculares AFLP e SSR; estimar a heterose e a capacidade de combinação das linhagens e verificar se a distância genética entre as linhagens obtidas por marcadores moleculares é correlacionada com a heterose dos híbridos simples.

\section{MATERIAL E MÉTODOS}

As linhagens utilizadas nos cruzamentos pertencem ao banco de germoplasma do IAC e se distinguem em dois grandes grupos: linhagens antigas do programa de melhoramento de milho e mais recentes, provenientes do Centro Internacional de Melhoramento de Milho e Trigo (CIMMYT) (Tabela 1).

Essas linhagens haviam sido caracterizadas quanto à divergência genética através de marcadores moleculares do tipo AFLP (Oliveira et al., 2004) e também pela associação dos marcadores AFLP e SSR (LABORDA et al., 2005) pelo Centro de Biologia Molecular da UNICAMP (CEBMEG), para estudos de diversidade genética e subsidiar o programa de melhoramento de milho do IAC. Com base nos resultados das análises moleculares, linhagens de diferentes distâncias genéticas foram cruzadas em esquema dialélico parcial.

Os 36 híbridos simples resultantes de um dialelo parcial $6 \times 6$ foram avaliados no Centro Experimental Central do IAC, em Campinas e no Pólo Regional de Mococa - APTA; as 12 linhagens parentais foram avaliadas apenas em Campinas, na safra 2004/2005. Por esse motivo, são considerados somente os resultados de Campinas para as análises de correlação. $\mathrm{O}$ delineamento experimental utilizado foi o de blocos ao acaso com três repetições e duas testemunhas comerciais (IAC 8333 e DKB 350). Cada parcela foi constituída por duas linhas de $5 \mathrm{~m}$ espaçadas de $0,90 \mathrm{~m}$ entre linhas e de $0,20 \mathrm{~m}$ entre plantas, com o total de 50 plantas.

Tabela 1. Características das linhagens utilizadas no dialelo parcial de milho

\begin{tabular}{llccccc}
\hline Linhagem & Procedência & Origem & Floresc. Masc. & Cor do grão & Tipo do grão & Grupo de Diversidade $\left(^{1}\right)$ \\
\hline L 8 & & dias & & \\
VER 266 & CIMMYT & Pop. 28 & 61 & Amarelo & Dentado & II \\
L 161 & IAC & Tuxpeno & 69 & Amarelo & Dentado & III \\
PM 624 & CIMMYT & Pop. 26 & 53 & Laranja & Semiduro & I \\
PM 684 & IAC & Tuxpeno & 69 & Amarelo & Dentado & IV \\
AL 745 & IAC & Tuxpeno & 64 & Amarelo & Dentado & II \\
L 3 & IAC & Cateto & 65 & Laranja & Duro & III \\
L 105 & CIMMYT & Pop. 24 & 60 & Amarelo & Semiduro & II \\
L 165 & CIMMYT & Pop. 26 & 57 & Laranja & Semiduro & I \\
IP 330 & CIMMYT & Pop. 27 & 63 & Laranja & Duro & I \\
L 157 & IAC & Cateto & 61 & Laranja & Semiduro & I \\
IP 398 & CIMMYT & Pop. 27 & 61 & Laranja & Duro & I
\end{tabular}

$\left({ }^{1}\right)$ Oliveira et al., 2004. 
Foram avaliados os seguintes caracteres agronômicos: altura da planta (AP), altura da espiga (AE) e massa de grãos (MG). Para as estimativas de parâmetros genéticos, os dados de massa de grãos foram corrigidos para umidade de $14 \%$ e estande ideal (CRuz e RegazzI, 1994). Realizaram-se análises da variância preliminares individuais e para a análise dialélica parcial foi utilizado o método de GERALDI e Miranda Filho (1988) proposto como adaptação do modelo 4 de GrifFING (1956) para dialelos parciais. As análises foram efetuadas com o Programa Genes (CRUZ, 1997).

As estimativas da heterose $(\mathrm{H})$ foram realizadas através de: $\mathrm{H}=\overline{\mathrm{F}}_{1}-\overline{M P}$, em que: $\overline{\mathrm{F}}_{1}=$ média do híbrido simples e $\overline{\mathrm{MP}}=$ média das linhagens parentais (FALCONER, 1981).

O DNA foi isolado de acordo com HoIsINGTON et al., (1994) e quantificadas em géis de agarose a 0,8\%. A amplificação dos SSRs seguiu um programa de PCR (Polymerase Chain Reaction) e a visualização dos produtos da PCR foi feita em géis agarose/metaphor $4 \%$, corados com brometo de etídeo. $\mathrm{Na}$ análise dos dados SSR, empregaram-se os programas Tools For Population Genetic Analyses (TFPGA) versão 1.3 (MilLer, 1997) e Numerical Taxonomy and Multivariate Analysis System (NTSYS) versão 2.1 (ROHLF, 1997).

A análise por AFLP foi realizada como descrita por Vos et al., (1995), utilizando o "AFLP Analysis Kit" (Life Technologies - GIBCO BRL). Os resultados SSR e AFLP foram interpretados através de um sistema binário, obtendo-se uma matriz utilizada para fins de cálculo de distância genética, feito com o auxílio do software NTSYS (Exeter Software, N.Y.; RoHLF, 1997), utilizando-se o coeficiente de similaridade de JACCARD (1908) e o método de agrupamento UPGMA. O coeficiente de similaridade (SG) foi convertido para distância genética (DG): DG = 1 - SG. Estimaram-se as correlações simples entre produtividade de grãos $(\mathrm{PG})$, heterose $(\mathrm{H})$ e capacidade específica de combinação $\left(\mathrm{s}_{\mathrm{ij}}\right)$ com a distância genética (DG) obtida por AFLP e SSR. O teste de MANTel (1967) foi utilizado, para testar a significância da associação entre as matrizes contendo diferentes tipos de distâncias entre pares de observações (SMOUSE et al., 1986; MANLY, 1991). Mil permutações aleatórias foram utilizadas para se testar a significância das correlações matriciais. O teste de Mantel simples foi realizado utilizando o pacote NTSYS (Numerical Taxonomy and Multivariate Analysis System).

\section{RESULTADOS E DISCUSSÃO}

Os quadrados médios das análises de variância individuais foram altamente significativos
$(\mathrm{P}<0,01)$ para o efeito de híbridos simples para todos caracteres avaliados, em ambos os locais. $\mathrm{Na}$ análise conjunta, houve interação genótipo por ambientes significativa $(\mathrm{P}<0,01)$ para massa de grãos.

Na tabela 2, verificam-se os valores médios de $\mathrm{AP}, \mathrm{AE}$, e MG dos híbridos simples experimentais e das duas testemunhas comerciais do dialelo, em Campinas e Mococa, constatando-se grande diversidade entre os híbridos experimentais, com MG variando de 3.738 a $9.516 \mathrm{~kg} \mathrm{ha}^{-1} \mathrm{em}$ Campinas e de 4.997 a $8.721 \mathrm{~kg} \mathrm{ha}^{-1} \mathrm{em}$ Mococa. O híbrido experimental que obteve maior produção em Campinas foi 3x1' (L161 x L3) e o híbrido 2x1' (VER266 x L3) o que mais se destacou no experimento em Mococa. De acordo com o dendograma obtido por OliveIRA et al. (2004), esses híbridos são formados pela combinação entre linhagens de grupos diferentes. No entanto, nenhum dos híbridos produzidos diferiu das testemunhas comerciais IAC 8333 e DKB 350, em relação a PG, pelo Teste de Tukey $(\mathrm{P}<0,05)$.

Os híbridos que se destacaram com MG superior a $8.000 \mathrm{~kg} \mathrm{ha}^{-1}$ e acima da média do experimento em Campinas foram: $2 \times 1$ ' (VER266 x L3), 2×2' (VER266 X L105), 2×5' (VER266 X L157), 3×1' (L161 x L3), 3×2' (L161 x L105), 4x1' (PM624 X L3), $4 \times 4^{\prime}$ (PM624 X IP330), 4×5' (PM624 X L157) e 4x6' (PM624 X IP398) (Tabela 2). Já em Mococa os híbridos que obtiveram produção de grãos superior a $7.000 \mathrm{~kg}$ ha $^{-1}$ foram: 2x1' (VER266 x L3), 2x2' (VER266 X L105),

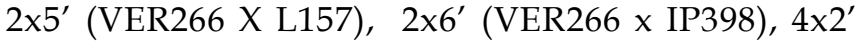
(PM624 X L105), 4x6' (PM624 X IP398), 5x1' (PM684 x L3), 5x2' (PM684 x L105) e 6x4' (AL745 x IP330) (Tabela 2). Observa-se que na maioria são híbridos de linhagens pertencentes a grupos heteróticos diferentes, exceto os híbridos $3 \times 2^{\prime}$ (Campinas), $5 \times 1^{\prime}$ e $6 \times 4^{\prime}$ (Mococa), formados por linhagens que pertencem ao mesmo grupo heterótico (Oliveira et al., 2004).

Em relação a $\mathrm{AP}$ e $\mathrm{AE}$, os híbridos produzidos ficaram com porte relativamente baixo, o que é uma característica desejável nas cultivares modernas de milho.

A análise de variância dos cruzamentos dialélicos evidenciou diferenças significativas $(\mathrm{P}<0,01)$ no quadrado médio de cruzamentos para todos os caracteres avaliados em Campinas e Mococa, o qual foi desdobrado em CGC e CEC (Tabela 3). Os quadrados médios referentes a CGC e CEC foram altamente significativos $(P<0,01)$ para $M G$, mas não significativos para a CEC referente a AP em Campinas e AE em Mococa. Ficou evidenciada maior magnitude da CGC para AP e AE e a importância da CGC e CEC para MG, comprovando a ação de efeitos aditivos e de dominância na manifestação da produtividade de grãos. 
Tabela 2. Valores médios de altura da planta (AP), altura da espiga (AE) e massa de grãos (MG) de 36 híbridos simples experimentais de milho (HS) resultantes do Dialelo e das testemunhas comerciais. Campinas e Mococa (SP), safra 2004/2005

\begin{tabular}{|c|c|c|c|c|c|c|c|}
\hline \multirow{2}{*}{ HS } & \multirow{2}{*}{ Origem } & \multicolumn{3}{|c|}{ Campinas } & \multicolumn{3}{|c|}{ Mococa } \\
\hline & & $\mathrm{AP}$ & $\mathrm{AE}$ & \multirow{3}{*}{$\begin{array}{c}\mathrm{MG} \\
\mathrm{kg} \mathrm{ha}^{-1} \\
6.602 \mathrm{~b}-\mathrm{d}\end{array}$} & \multirow{2}{*}{\multicolumn{2}{|c|}{$\begin{array}{ll}\mathrm{AP} & \mathrm{AE} \\
& \mathrm{cm}\end{array}$}} & \multirow{3}{*}{$\begin{array}{c}\text { MG } \\
\mathrm{kg} \mathrm{ha}^{-1} \\
5.399 \mathrm{c}-\mathrm{f}\end{array}$} \\
\hline \multirow[b]{2}{*}{$1 \times 1^{\prime}$} & \multirow[b]{2}{*}{ L8 X L3 } & \multicolumn{2}{|c|}{$\mathrm{cm}$} & & & & \\
\hline & & $2091-n$ & $135 \mathrm{~d}-\mathrm{i}$ & & $200 \mathrm{fg}$ & 108 e-i & \\
\hline $1 \times 2^{\prime}$ & L8 X L105 & $204 \mathrm{mn}$ & 115 h-1 & $7.445 \mathrm{a}-\mathrm{c}$ & $202 \mathrm{fg}$ & 109 e-i & $6.425 \mathrm{~b}-\mathrm{f}$ \\
\hline $1 \times 3^{\prime}$ & L8 X L165 & $2101-n$ & 104 kl & $6.459 \mathrm{~b}-\mathrm{d}$ & $216 \mathrm{~d}-\mathrm{g}$ & $106 \mathrm{~g}-\mathrm{i}$ & $6.175 \mathrm{~b}-\mathrm{f}$ \\
\hline $1 \times 4^{\prime}$ & L8 X IP330 & $217 \mathrm{j}-\mathrm{n}$ & $133 \mathrm{~d}-\mathrm{j}$ & $6.996 \mathrm{a}-\mathrm{c}$ & 209 e-g & 109 e-i & $6.386 \mathrm{~b}-\mathrm{f}$ \\
\hline $1 \times 5^{\prime}$ & L8 X L157 & $200 n$ & 110 i-1 & $7.614 \mathrm{a}-\mathrm{c}$ & $196 \mathrm{~g}$ & $100 \mathrm{i}$ & 5.171 ef \\
\hline $1 \times 6^{\prime}$ & L8 X IP398 & $228 \mathrm{~d}-\mathrm{m}$ & $140 \mathrm{c}-\mathrm{h}$ & $6.592 \mathrm{~b}-\mathrm{d}$ & $220 \mathrm{c}-\mathrm{g}$ & $127 \mathrm{c}-\mathrm{i}$ & $6.870 \mathrm{a}-\mathrm{f}$ \\
\hline $2 \times 1^{\prime}$ & VER266 X L3 & 233 c-1 & $145 \mathrm{~b}-\mathrm{g}$ & $8.244 \mathrm{a}-\mathrm{c}$ & $249 \mathrm{a}-\mathrm{g}$ & 148 a-f & $7.378 \mathrm{a}-\mathrm{c}$ \\
\hline $2 \times 2^{\prime}$ & VER266 X L105 & $2081-n$ & $125 \mathrm{~g}-1$ & $8.068 \mathrm{a}-\mathrm{c}$ & $234 \mathrm{~b}-\mathrm{g}$ & $142 \mathrm{~b}-\mathrm{h}$ & 7.031 a-e \\
\hline $2 \times 3^{\prime}$ & VER266 X L165 & $242 \mathrm{~b}-\mathrm{j}$ & $143 \mathrm{~b}-\mathrm{g}$ & $7.181 \mathrm{a}-\mathrm{c}$ & $246 \mathrm{a}-\mathrm{g}$ & 148 a-f & $6.642 \mathrm{~b}-\mathrm{f}$ \\
\hline $2 \times 4^{\prime}$ & VER266 X IP330 & $237 \mathrm{~b}-\mathrm{k}$ & $140 \mathrm{c}-\mathrm{h}$ & 7.416 a-c & $247 \mathrm{a}-\mathrm{g}$ & $146 \mathrm{a}-\mathrm{g}$ & $6.368 \mathrm{~b}-\mathrm{f}$ \\
\hline $2 \times 5^{\prime}$ & VER266 X L157 & $238 \mathrm{~b}-\mathrm{k}$ & 138 d-h & $8.301 \mathrm{a}-\mathrm{c}$ & 255 a-f & $138 \mathrm{~b}-\mathrm{h}$ & $7.330 \mathrm{a}-\mathrm{c}$ \\
\hline $2 \times 6^{\prime}$ & VER266 X IP398 & 258 a-c & $166 \mathrm{ab}$ & $7.557 \mathrm{a}-\mathrm{c}$ & $266 \mathrm{a}-\mathrm{d}$ & 156 a-d & $7.267 \mathrm{a}-\mathrm{d}$ \\
\hline $3 \times 1^{\prime}$ & L161 X L3 & 221 h-n & $128 \mathrm{f}-1$ & $8.602 \mathrm{ab}$ & $222 \mathrm{c}-\mathrm{g}$ & $121 \mathrm{~d}-\mathrm{i}$ & $5.766 \mathrm{~b}-\mathrm{f}$ \\
\hline $3 \times 2^{\prime}$ & L161 X L105 & 221 h-n & $131 \mathrm{f}-\mathrm{j}$ & 8.248 a-c & $197 \mathrm{~g}$ & $116 \mathrm{~d}-\mathrm{i}$ & $6.527 b-f$ \\
\hline $3 \times 3^{\prime}$ & L161 X L165 & 217 j-n & $127 \mathrm{f}-1$ & $6.268 \mathrm{~b}-\mathrm{d}$ & $199 \mathrm{fg}$ & 108 e-i & $5.938 \mathrm{~b}-\mathrm{f}$ \\
\hline $3 \times 4^{\prime}$ & L161 X IP330 & 238 b-k & $140 \mathrm{c}-\mathrm{h}$ & $7.676 \mathrm{a}-\mathrm{c}$ & $227 \mathrm{~b}-\mathrm{g}$ & $123 \mathrm{~d}-\mathrm{i}$ & $6.582 \mathrm{~b}-\mathrm{f}$ \\
\hline $3 \times 5^{\prime}$ & L161 X L157 & 222 g-n & $122 \mathrm{~g}-1$ & $6.226 \mathrm{~b}-\mathrm{d}$ & $201 \mathrm{fg}$ & $105 \mathrm{~g}-\mathrm{i}$ & $6.284 b-f$ \\
\hline $3 \times 6^{\prime}$ & L161 X IP398 & 219 i-n & 132 e-j & $6.104 \mathrm{~b}-\mathrm{d}$ & $212 \mathrm{~d}-\mathrm{g}$ & $126 \mathrm{c}-\mathrm{i}$ & $6.687 b-f$ \\
\hline $4 \times 1^{\prime}$ & PM624 X L3 & $237 \mathrm{~b}-\mathrm{k}$ & $151 \mathrm{a}-\mathrm{f}$ & 8.317 a-c & $234 \mathrm{~b}-\mathrm{g}$ & $140 \mathrm{~b}-\mathrm{h}$ & $6.725 \mathrm{a}-\mathrm{f}$ \\
\hline $4 \times 2^{\prime}$ & PM624 X L105 & $223 \mathrm{f}-\mathrm{n}$ & $134 \mathrm{~d}-\mathrm{i}$ & $7.338 \mathrm{a}-\mathrm{c}$ & 254 a-f & 143 a-h & $7.267 \mathrm{a}-\mathrm{d}$ \\
\hline $4 \times 3^{\prime}$ & PM624 X L165 & $256 a-c$ & 158 a-d & $6.929 \mathrm{a}-\mathrm{c}$ & $244 \mathrm{a}-\mathrm{g}$ & 134 b-h & $5.499 \mathrm{c}-\mathrm{f}$ \\
\hline $4 \times 4^{\prime}$ & PM624 X IP330 & 249 a-f & 151 a-f & $8.028 \mathrm{a}-\mathrm{c}$ & $219 \mathrm{~d}-\mathrm{g}$ & $126 \mathrm{c}-\mathrm{i}$ & $6.349 \mathrm{~b}-\mathrm{f}$ \\
\hline $4 \times 5^{\prime}$ & PM624 X L157 & $243 \mathrm{~b}-\mathrm{j}$ & 138 d-h & $8.301 \mathrm{a}-\mathrm{c}$ & $222 \mathrm{c}-\mathrm{g}$ & $136 \mathrm{~b}-\mathrm{h}$ & $6.445 \mathrm{~b}-\mathrm{f}$ \\
\hline $4 \times 6^{\prime}$ & PM624 X IP398 & $261 \mathrm{ab}$ & 165 a-c & $8.733 \mathrm{ab}$ & $266 a-d$ & $165 \mathrm{a}-\mathrm{c}$ & $7.289 \mathrm{a}-\mathrm{d}$ \\
\hline $5 \times 1^{\prime}$ & PM684 X L3 & 250 a-e & $152 \mathrm{a}-\mathrm{f}$ & $3.738 \mathrm{~d}$ & $276 \mathrm{a}-\mathrm{c}$ & 149 a-e & $7.377 \mathrm{a}-\mathrm{c}$ \\
\hline $5 \times 2^{\prime}$ & PM684 X L105 & $257 \mathrm{a}-\mathrm{c}$ & 157 a-e & $5.573 \mathrm{~cd}$ & 263 a-e & 157 a-d & $7.336 \mathrm{a}-\mathrm{c}$ \\
\hline $5 \times 3^{\prime}$ & PM684 X L165 & $247 \mathrm{~b}-\mathrm{h}$ & 139 d-h & $5.686 \mathrm{~cd}$ & $252 \mathrm{a}-\mathrm{g}$ & $123 \mathrm{~d}-\mathrm{i}$ & $6.918 \mathrm{a}-\mathrm{f}$ \\
\hline $5 \times 4^{\prime}$ & PM684 X IP330 & $261 \mathrm{ab}$ & $166 \mathrm{ab}$ & $7.315 \mathrm{a}-\mathrm{c}$ & $279 \mathrm{ab}$ & $169 \mathrm{ab}$ & $6.518 \mathrm{~b}-\mathrm{f}$ \\
\hline $5 \times 5^{\prime}$ & PM684 X L157 & $252 \mathrm{a}-\mathrm{d}$ & $147 \mathrm{~b}-\mathrm{g}$ & $6.784 \mathrm{a}-\mathrm{c}$ & 262 a-e & 143 a-h & $6.583 \mathrm{~b}-\mathrm{f}$ \\
\hline $5 \times 6^{\prime}$ & PM684 X IP398 & $275 \mathrm{a}$ & $176 \mathrm{a}$ & $5.568 \mathrm{~cd}$ & $293 \mathrm{a}$ & $184 \mathrm{a}$ & $6.816 \mathrm{a}-\mathrm{f}$ \\
\hline $6 \times 1^{\prime}$ & AL745 X L3 & $234 c-1$ & $125 \mathrm{~g}-1$ & $7.678 \mathrm{a}-\mathrm{c}$ & $226 b-g$ & $121 \mathrm{~d}-\mathrm{i}$ & $5.906 \mathrm{~b}-\mathrm{f}$ \\
\hline $6 \times 2^{\prime}$ & AL745 X L105 & 225 e-n & $128 \mathrm{f}-1$ & $7.995 \mathrm{a}-\mathrm{c}$ & 239 a-g & $129 \mathrm{~b}-\mathrm{i}$ & $6.583 \mathrm{~b}-\mathrm{f}$ \\
\hline $6 \times 3^{\prime}$ & AL745 X L165 & $248 \mathrm{~b}-\mathrm{g}$ & $129 \mathrm{f}-\mathrm{k}$ & $6.123 \mathrm{~cd}$ & $227 \mathrm{~b}-\mathrm{g}$ & 102 hi & $4.997 \mathrm{f}$ \\
\hline $6 \times 4^{\prime}$ & AL745 X IP330 & 249 a-f & $151 \mathrm{a}-\mathrm{f}$ & $7.513 \mathrm{a}-\mathrm{c}$ & $252 \mathrm{a}-\mathrm{g}$ & $134 \mathrm{~b}-\mathrm{h}$ & 7.123 a-e \\
\hline $6 \times 5^{\prime}$ & AL745 X L157 & 229 d-m & $133 \mathrm{~d}-\mathrm{j}$ & $6.939 \mathrm{a}-\mathrm{c}$ & $216 \mathrm{~d}-\mathrm{g}$ & $114 \mathrm{e}-\mathrm{i}$ & $6.242 \mathrm{~b}-\mathrm{f}$ \\
\hline \multirow[t]{3}{*}{$6 \times 6^{\prime}$} & AL745 X IP398 & $245 \mathrm{~b}-\mathrm{i}$ & 143 b-j & $6.113 \mathrm{~b}-\mathrm{d}$ & 249 a-g & 139 b-h & $5.302 \mathrm{~d}-\mathrm{f}$ \\
\hline & DKB 350 & 213 k-n & 108 j-1 & $7.965 a-c$ & $199 \mathrm{fg}$ & 104 hi & $7.670 \mathrm{ab}$ \\
\hline & IAC 8333 & $199 \mathrm{n}$ & 1031 & $9.516 \mathrm{a}$ & $197 \mathrm{~g}$ & $107 \mathrm{f}-\mathrm{i}$ & 8.721 a \\
\hline Média & - & 221 & 128 & 7.204 & 233 & 130 & 6.576 \\
\hline Dms (5\%) & - & 26,72 & 25,39 & 2898 & 56,22 & 41,10 & 2025 \\
\hline CV $(\%)$ & - & 3,5 & 5,6 & 12,2 & 7,3 & 9,6 & 9,4 \\
\hline
\end{tabular}

Médias com letras diferentes na coluna diferem a $5 \%$ pelo Teste de Tukey. 
Tabela 3. Quadrados médios da análise de variância dialélica conjunta de altura da planta (AP), altura da espiga (AE) e massa de grãos (MG) de 36 híbridos simples experimentais de milho. Campinas e Mococa (SP), safra 2004/2005

\begin{tabular}{|c|c|c|c|c|c|c|c|}
\hline \multirow{3}{*}{ Fonte de Variação } & \multirow{3}{*}{ G.L. } & \multicolumn{6}{|c|}{ Quadrados Médios } \\
\hline & & \multicolumn{3}{|c|}{ Campinas } & \multicolumn{3}{|c|}{ Mococa } \\
\hline & & $\mathrm{AP}$ & $\mathrm{AE}$ & MG & $\mathrm{AP}$ & $\mathrm{AE}$ & MG \\
\hline & & \multicolumn{2}{|c|}{$\mathrm{m}$} & t.ha ${ }^{-1}$ & \multicolumn{2}{|c|}{$\mathrm{m}$} & t.ha ${ }^{-1}$ \\
\hline Cruzamentos & 35 & $0,102^{* *}$ & $0,077^{* *}$ & $2,804^{* *}$ & $0,282^{* *}$ & $0,124^{* *}$ & $1,294^{* *}$ \\
\hline CGC 1 & 5 & $0,467^{* *}$ & $0,280 * *$ & $8,832^{* *}$ & $1,23 * *$ & $0,569 * *$ & $3,209 * *$ \\
\hline CGC 2 & 5 & $0,141^{* *}$ & $0,151^{* *}$ & $2,632 * *$ & $0,181^{* *}$ & $0,195^{* *}$ & $1,539 * *$ \\
\hline CEC $1 \times 2$ & 25 & 0,021 & $0,021^{*}$ & $1,633^{* *}$ & $0,113^{* *}$ & 0,0213 & $0,861^{* *}$ \\
\hline Resíduo & 94 & 0,022 & 0,011 & 0,591 & 0,0305 & 0,016 & $0,366^{* *}$ \\
\hline Média & 2,35 & 1,39 & 6471 & 2,35 & 1,31 & 6486 & - \\
\hline
\end{tabular}

*;** significativo a $5 \%$ e $1 \%$ pelo Teste $\mathrm{F}$.

Conforme relataram COMSTOCK E ROBINSON (1948) e GARDNER et al., (1953), nos caracteres AP e AE há predominância de efeitos aditivos e dominância apenas parcial. A predominância da ação gênica aditiva no controle da produtividade de grãos foi também verificada por SANTOS et al. (1985); GAMA et al., (1995); Pinto et al., (2001) e SCAPIM et al. (2002).

Os efeitos da capacidade geral de combinação das linhagens para MG (Tabela 4), mostraram valores positivos em ambos os locais para a VER266 e PM624 do Grupo 1 e L105 e IP330 do Grupo 2.

Tabela 4. Estimativas dos efeitos da capacidade geral de combinação $\left(\mathrm{g}_{\mathrm{i}}\right)$, de 12 linhagens de milho do Dialelo, para massa de grãos (MG). Campinas e Mococa (SP), safra 2004/2005

\begin{tabular}{llll}
\hline \multirow{2}{*}{ Linhagens } & \multicolumn{3}{c}{$\hat{\mathrm{g}}_{\mathrm{i}}(\mathrm{MG})$} \\
\cline { 2 - 4 } & Campinas & Mococa & Conjunta \\
\hline & & $\mathrm{kg} \mathrm{ha}^{-1}$ & \\
\end{tabular}

Grupo 1

1 L8

2 VER266

$-166,805$

676,361

$-415,139$

$-290,972$

3 L161

68,528

516,528

596,445

4 PM624

822,028

$-188,805$

$-60,139$

5 PM684

$-1341,472$

109,528

465,778

438,528

$-451,472$

6 AL745

$-58,639$

$-460,639$

$-259,639$

Grupo 2

1' L3

78,694

2' L105

325,694

3' L165

$-677,972$

$-60,972$

375,361

8,861

4' IP330

371,861

5' L157

242,361

6' IP398

$-340,639$

191,027
$-457,972$

68,194

$-143,639$

219,028

130,196
As médias de massa de grãos (MG), heterose em relação à média dos pais $(\mathrm{H})$, estimativa da capacidade específica $\left(\mathrm{s}_{\mathrm{ij}}\right)$ e os valores de divergência genética (DG) com base em AFLP e em SSR, respectivamente, somente para o dialelo em Campinas, estão apresentadas na tabela 5.

As estimativas de heterose em relação à média dos pais $(\mathrm{H})$ variaram de -559 a $6.320 \mathrm{~kg} \mathrm{ha}^{-1}$, confirmando-se a elevada variabilidade genética entre os híbridos e a grande diversidade entre as linhagens parentais. O híbrido 4x6' (PM624 x IP398) obteve maior valor de heterose, revelando a importância do emprego da combinação entre linhagens de grupos heteróticos divergentes. O híbrido 5x1' (PM684 x L3) obteve o menor valor de heterose, por ser formado pela combinação de linhagens pertencentes ao mesmo grupo de divergência genética.

Em relação à estimativa da capacidade específica de combinação para MG, destacaram-se: 3x1' (L161 x L13), 3×2' (L161 x L105), 4x6' (PM624 x IP398), 5x4' (PM684 x IP330) e 5x5' (PM684 x L157), obtendo valores $1.337 ; 735 ; 1.133 ; 1.166$ e de $764 \mathrm{~kg} \mathrm{ha}^{-1}$.

As distâncias genéticas variaram de 0,41 a 0,56 para o marcador AFLP, enquanto as distâncias baseadas em SSR no mesmo ensaio, variaram de 0,60 a 0,82, demonstrando maior amplitude de variação através de SSR. Para os dados embasados em AFLP, os cruzamentos que obtiveram menores coeficientes de distância genética foram $2 \times 2^{\prime}(0,41), 2 \times 5^{\prime}(0,41), 2 \times 6^{\prime}$ $(0,43), 2 \times 3^{\prime}(0,43)$ e $2 \times 4^{\prime}(0,44)$. Verifica-se que apesar dessas linhagens envolvidas nesses cruzamentos estarem separadas em grupos diferentes, elas podem provavelmente possuir origens semelhantes. Os valores mais elevados de distância foram observados nos cruzamentos com a linhagem 6 (AL745): $6 \times 11^{\prime}$ $(0,56), 6 \times 4^{\prime}(0,54), 6 \times 5^{\prime}(0,54), 6 \times 3^{\prime}(0,53)$ e também o cruzamento $1 \times 6^{\prime}(0,55)$. 
Tabela 5. Valores médios de massa de grãos (MG) de 36 híbridos simples de milho do Dialelo, heterose em relação a média dos pais $(\mathrm{H})$, estimativas da capacidade específica de combinação $\left(\hat{\mathrm{s}}_{\mathrm{ij}}\right)$ e valores de divergência genética (DG) entre linhagens de milho, obtida através de marcadores moleculares AFLP e SSR. Campinas (SP), safra 2004/2005

\begin{tabular}{|c|c|c|c|c|c|c|}
\hline Híbridos & Origem & PG & $\mathrm{H}$ & $\hat{\mathrm{s}}_{\mathrm{ij}}$ & DG AFLP & DG SSR \\
\hline & & 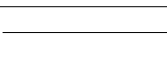 & $\mathrm{kg} \mathrm{ha}^{-1}$ & +2 & & \\
\hline $1 \times 1^{\prime}$ & L8 X L3 & $6.602 \mathrm{~b}-\mathrm{d}$ & 3.064 & $-427,528$ & 0,5125 & 0,7222 \\
\hline $1 \times 2^{\prime}$ & L8 X L105 & $7.445 \mathrm{a}-\mathrm{c}$ & 3.938 & 167,472 & 0,4748 & 0,8133 \\
\hline $1 \times 3^{\prime}$ & L8 X L165 & $6.459 \mathrm{~b}-\mathrm{d}$ & 3.219 & 184,139 & 0,4724 & 0,7703 \\
\hline $1 \times 4^{\prime}$ & L8 X IP330 & $6.996 \mathrm{a}-\mathrm{c}$ & 3.448 & $-327,694$ & 0,4837 & 0,7671 \\
\hline $1 \times 5^{\prime}$ & L8 X L157 & $7.614 \mathrm{a}-\mathrm{c}$ & 4.379 & 421,806 & 0,4368 & 0,8072 \\
\hline $1 \times 6^{\prime}$ & L8 X IP398 & $6.592 \mathrm{~b}-\mathrm{d}$ & 3.330 & $-18,194$ & 0,5478 & 0,7922 \\
\hline $2 \times 1^{\prime}$ & VER266 X L3 & $8.244 \mathrm{a}-\mathrm{c}$ & 5.760 & 371,306 & 0,4845 & - \\
\hline $2 \times 2^{\prime}$ & VER266 X L105 & 8.068 a-c & 5.614 & $-51,694$ & 0,4141 & - \\
\hline $2 \times 3^{\prime}$ & VER266 X L165 & $7.181 \mathrm{a}-\mathrm{c}$ & 4.995 & 63,972 & 0,4352 & - \\
\hline $2 \times 4^{\prime}$ & VER266X IP330 & 7.416 a-c & 4.922 & $-750,861$ & 0,4358 & - \\
\hline $2 \times 5^{\prime}$ & VER266 X L157 & $8.301 \mathrm{a}-\mathrm{c}$ & 6.120 & 263,639 & 0,4143 & - \\
\hline $2 \times 6^{\prime}$ & VER266X IP398 & 7.557 a-c & 5.349 & 103,639 & 0,4322 & - \\
\hline $3 \times 1^{\prime}$ & L161 X L3 & $8.602 \mathrm{ab}$ & 3.334 & 1337,139 & 0,4740 & 0,7108 \\
\hline $3 \times 2^{\prime}$ & L161 X L105 & 8.248 a-c & 3.010 & 735,139 & 0,4886 & 0,7470 \\
\hline $3 \times 3^{\prime}$ & L161 X L165 & $6.268 \mathrm{~b}-\mathrm{d}$ & 1.297 & $-242,194$ & 0,4516 & 0,6579 \\
\hline $3 \times 4^{\prime}$ & L161 X IP330 & 7.676 a-c & 2.397 & 115,972 & 0,4755 & 0,6835 \\
\hline $3 \times 5^{\prime}$ & L161 X L157 & $6.226 \mathrm{~b}-\mathrm{d}$ & 1.261 & $-1203,530$ & 0,4462 & 0,6941 \\
\hline $3 \times 6^{\prime}$ & L161 X IP398 & $6.104 \mathrm{~b}-\mathrm{d}$ & 1.111 & $-742,528$ & 0,4805 & 0,6914 \\
\hline $4 \times 1^{\prime}$ & PM624 X L3 & $8.317 \mathrm{a}-\mathrm{c}$ & 5.629 & 297,639 & 0,5161 & 0,7750 \\
\hline $4 \times 2^{\prime}$ & PM624 X L105 & $7.338 \mathrm{a}-\mathrm{c}$ & 4.680 & $-928,361$ & 0,4316 & 0,7632 \\
\hline $4 \times 3^{\prime}$ & PM624 X L165 & $6.929 \mathrm{a}-\mathrm{c}$ & 4.538 & $-333,694$ & 0,5259 & 0,7123 \\
\hline $4 \times 4^{\prime}$ & PM624 X IP330 & $8.028 \mathrm{a}-\mathrm{c}$ & 5.329 & $-285,528$ & 0,4943 & 0,7027 \\
\hline $4 \times 5^{\prime}$ & PM624 X L157 & $8.301 \mathrm{a}-\mathrm{c}$ & 5.916 & 116,972 & 0,4929 & 0,8182 \\
\hline $4 \times 6^{\prime}$ & PM624 X IP398 & $8.733 \mathrm{ab}$ & 6.320 & 1132,972 & 0,5072 & 0,7750 \\
\hline $5 \times 1^{\prime}$ & PM684 X L3 & $3.738 \mathrm{~d}$ & -0.559 & $-2117,860$ & 0,5121 & 0,7848 \\
\hline $5 \times 2^{\prime}$ & PM684 X L105 & $5.573 \mathrm{~cd}$ & 1.306 & $-530,861$ & 0,4595 & 0,8205 \\
\hline $5 \times 3^{\prime}$ & PM684 X L165 & $5.686 \mathrm{~cd}$ & 1.686 & 586,806 & 0,4692 & 0,7397 \\
\hline $5 \times 4^{\prime}$ & PM684 X IP330 & $7.315 \mathrm{a}-\mathrm{c}$ & 3.007 & 1165,972 & 0,5028 & 0,7297 \\
\hline $5 \times 5^{\prime}$ & PM684 X L157 & $6.784 \mathrm{a}-\mathrm{c}$ & 2.790 & 764,472 & 0,4674 & 0,7375 \\
\hline $5 \times 6^{\prime}$ & PM684 X IP398 & $5.568 \mathrm{~cd}$ & 1.546 & 131,472 & 0,4974 & 0,7500 \\
\hline $6 \times 1^{\prime}$ & AL745 X L3 & $7.678 \mathrm{a}-\mathrm{c}$ & 5.140 & 539,306 & 0,5655 & 0,7105 \\
\hline $6 \times 2^{\prime}$ & AL745 X L105 & $7.995 \mathrm{a}-\mathrm{c}$ & 5.487 & 608,306 & 0,4918 & 0,6944 \\
\hline $6 \times 3^{\prime}$ & AL745 X L165 & $6.123 \mathrm{~cd}$ & 3.882 & $-259,028$ & 0,5275 & 0,7949 \\
\hline $6 \times 4^{\prime}$ & AL745 X IP330 & $7.513 \mathrm{a}-\mathrm{c}$ & 4.964 & 82,139 & 0,5414 & 0,5970 \\
\hline $6 \times 5^{\prime}$ & AL745 X L157 & $6.939 \mathrm{a}-\mathrm{c}$ & 4.704 & $-363,361$ & 0,5376 & 0,7619 \\
\hline \multirow[t]{3}{*}{$6 \times 6^{\prime}$} & AL745 X IP398 & $6.113 \mathrm{~b}-\mathrm{d}$ & 3.850 & $-607,361$ & 0,5070 & 0,6575 \\
\hline & DKB 350 & $7.965 \mathrm{a}-\mathrm{c}$ & - & - & - & - \\
\hline & IAC 8333 & $9.516 \mathrm{a}$ & - & - & - & - \\
\hline Média & & 7.204 & - & - & - & - \\
\hline DMS (5\%) & & 2898 & - & - & - & - \\
\hline CV $(\%)$ & & 12,2 & - & - & - & - \\
\hline
\end{tabular}

Médias com letras diferentes na coluna diferem a 5\% pelo Teste de Tukey. 
Para as análises de divergência por SSR, os menores valores foram observados para os cruzamentos envolvendo linhagens pertencentes a grupos heteróticos e origens diferentes, como $6 \times 44^{\prime}$ $(0,60)$ e $6 \times 66^{\prime}(0,66)$. Já os maiores valores observados para DG foram obtidos pelos cruzamentos $5 \times 22^{\prime}(0,82)$, $4 \times 5^{\prime}(0,82), 1 \times 2^{\prime}(0,81)$ e $1 \times 5^{\prime}(0,81)$, mostrando que as linhagens pertencem a grupos heteróticos diferentes.

$\mathrm{Na}$ tabela 6 estão apresentados os valores da correlação entre as distâncias genéticas (DG) obtidas por marcadores AFLP e SSR, com produtividade $(\mathrm{MG})$, heterose $(\mathrm{H})$ e capacidade específica de combinação $\left(\mathrm{s}_{\mathrm{ij}}\right)$.

Tabela 6. Estimativas dos coeficientes de correlação simples (r) entre massa de grãos (MG), heterose em relação à média dos pais $(\mathrm{H})$ e capacidade específica de combinação $\left(\hat{s}_{\mathrm{ij}}\right)$ (Griffing, 1956) com divergência genética (DG) por AFLP e SSR, em um dialelo 6x6 de milho. Campinas (SP), safra 2004/2005.

\begin{tabular}{lccc}
\hline & DG AFLP & DG SSR \\
\hline PG & $0,16^{\text {ns }}$ & $0,14^{\text {ns }}$ \\
$\mathrm{H}$ & $0,00^{\text {ns }}$ & & $-0,03^{\text {ns }}$ \\
$\hat{\mathrm{s}}_{\mathrm{ij}}$ & $0,09^{\mathrm{ns}}$ & $0,10^{\mathrm{ns}}$ \\
\hline AFLP & & \\
& & SSR & \\
\hline
\end{tabular}

** : significativo a $1 \%$ pelo teste de Mantel; ns = não significativo

Os valores das correlações entre DG obtida por AFLP, e as médias de produtividade dos híbridos simples, foram positivos porém não significativos pelo teste de Mantel $(r=0,16)$. Isto pode ser explicado pela ausência de dominância, pelo fato de as marcas não estarem associadas a um ou mais QTLs determinantes do caráter em questão e/ou devido à produtividade não possuir alta herdabilidade (BERNARDO, 1992).

Semelhantes resultados foram constatados pelas correlações de DG obtidas por marcadores SSR com produtividade $(\mathrm{r}=0,14)$. MELCHINGER et al., (1990), Melchinger et al., (1992) e Charcosset e Essioux (1994) também observaram que não houve correlação entre divergência genética e produtividade dos híbridos produzidos da combinação entre linhagens de grupos heteróticos diferentes.

Não houve correlação entre DG e heterose, revelando que o valor foi baixo para as distâncias baseadas em AFLP $(r=0,00)$ e negativa para SSR $(r=$ $-0,03$ ). Esses resultados discordam de Benchimol et al. (2000) e ReIf et al. (2003), que obtiveram correlação positiva e significativa entre divergência genética e heterose em populações contrastantes, mas concordam com Melo et al. (2001), que não obtiveram tal correlação, utilizando genitores comerciais de milho.

Resultados interessantes sobre correlações entre divergência genética de linhagens e produtividade dos híbridos foram verificados por BARBOSA et al. (2003), com dois grupos de linhagens derivadas das populações BR 105 e BR 106: os coeficientes de correlação foram altos para cruzamentos entre BR106xBR106 (0,91 e 0,82 para AFLP e SSR respectivamente); moderados para cruzamentos entre BR105xBR105 $(0,52)$ e baixos entre cruzamentos de linhagens de BR105xBR106 (0,29 e 0,16 para AFLP e SSR respectivamente). Assim, parece haver maior sucesso na predição de cruzamentos a partir de distâncias genéticas entre linhagens dentro de uma mesma população de milho. A baixa correlação em nível interpopulacional pode ser decorrente da menor variação de distância genética, provavelmente causada por seleção prévia para capacidade combinatória.

Foram observados baixos valores para as correlações entre DG obtida por ambos os marcadores e capacidade específica de combinação. $\mathrm{O}$ valor foi de $\mathrm{r}=0,09$ para AFLP e $\mathrm{r}=0,10$ para SSR. Esses resultados corroboram os de Mool et al. (1965), CRESS (1966) e Melo (2001), quando relatam que o fato de alguns parentais possuírem altos níveis de distância genética não garante que no cruzamento entre eles haverá alta heterose, resultando em altos valores de CEC.

A correlação entre os dois marcadores moleculares empregados neste estudo obteve valor elevado e significativo $r=0,991^{* *}(P<0,01)$, mostrando que o emprego desses marcadores moleculares foi útil e consistente nas análises da divergência genética das linhagens parentais.

\section{CONCLUSÕES}

1. As linhagens que se destacaram quanto à capacidade geral de combinação para massa de grãos foram VER266, PM624, L105 e IP330.

2. Houve predominância dos efeitos de aditividade e de dominância para $M G$, o que demonstra a importância da CGC e da CEC em um programa de melhoramento de milho híbrido.

3. As distâncias genéticas obtidas por AFLP e SSR não foram correlacionadas com a heterose dos híbridos, não permitindo inferir sobre os cruzamentos em programas de híbridos. 
4. De maneira geral, altas estimativas de divergência genética entre as linhagens não implicaram altos valores de CEC e não se correlacionaram com a produtividade do híbrido. Portanto, não é possível predizer sobre a CEC e produtividade de grãos nos cruzamentos baseando-se na divergência genética entre as linhagens envolvidas.

\section{AGRADECIMENTOS}

À FAPESP - pelo financiamento do projeto. À pesquisadora Maria Imaculada Zucchi, pela ajuda e interpretação das estatísticas de Mantel.

\section{REFERÊNCIAS}

BARBOSA, A.M.M.; GERALDI, I.O.; BENCHIMOL,L.L.; GARCIA, A.A.F.; SOUZA, C.L.; SOUZA, A.P. Relationship of intra- and interpopulation tropical maize single cross hybrid performance and genetic distances computed from AFLP and SSR markers. Euphytica, Netherlands, v.130, p.87-99, 2003.

BENCHIMOL, L.L.; SOUZA JÚNIOR C.L.; GARCIA, A.A.F; KONO, P.M.S.; MANGOLIN, C.A.; BARBOSA, A..M..M.; COELHO, A.S.G.; SOUZA, A.P. Genetic diversity in tropical maize inbred lines: heterotic group assignment and hybrid performance determined by RFLP markers. Plant Breeding, Berlin, v.119, p.491-496, 2000.

BERNARDO, R. Relationship between single-cross performance and molecular marker heterozygosity. Theoretical and Applied Genetics, Berlin, v.83, p.628-634, 1992.

CHARCOSSET, A.; ESSIOUX, L. The effect of population struture on the relationship between heterosis and heterozygosity at marker loci. Theoretical and Applied Genetics, Berlin, v.89, n.2-3, p.336-343, 1994.

COMSTOCK, R.E.; ROBINSON, H.F. The components of genetic variance in populations of biparental progenies and their use in estimating the average degree of dominance. Biometrics, Alexandria, v.4, p.254-266, 1948.

CRESS, C.E. Heterosis of hybrid related to gene frequence differences between two populations. Genetics, New York, v.53, n.3, p.269-274, Feb. 1966.

CRUZ, C.D. Aplicativo computacional em Genética e Estatística: Programa Genes. Viçosa: UFV, 1997. 442 p.

CRUZ, C.D.; REGAZZI, A.J. Modelos biométricos aplicados ao melhoramento genético. Viçosa, UFV, 1997. 380p.

FALCONER, D.S. Introduction to quantitative genetics. 2.ed. London: Longman, 1981.

GADHERI, A.; ADAMS, M.W.; NASSIB, A.M. Relationship between genetic distance and heterosis for yield and morphological traits in dry Edible Bean and Faba Bean. Crop Science, Madison, v.24, p.37-42, 1984.
GAMA, E.E.G.; HALLAUER A.R.; FERRÃO, R.G. BARBOSA, D.M. Heterosis in maize single crosses derived from a yellow Tuxpeño variety in Brazil. Revista Brasileira de Genética, Ribeirão Preto, v.18, p.81-85, 1995.

GARDNER, C.O.; HARVEY, P.H.; COMSTOCK, R.E. Dominance of genes controlling quantitative characters in maize. Biometrics, Alexandria, v.45, p.186-191, 1953.

GERALDI, I.O. e MIRANDA FILHO, J.B. Adapted models for the analysis of combining abilty of varieties in partial diallel crosses. Revista Brasileira de Genética, Ribeirão Preto, v.11, n.2, p.419-30, 1988.

GRIFFING, J.B. A generalized treatment of the use of diallel crosses in quantitative inheritance. Heredity, Harlow, v.10, p. 31-50, 1956a.

GRIFFING, J.B. Concept of general and specific combining ability in relation to diallel systems. Australian Journal of Biological Science, East Melbourne, v.9, p. 463-493, 1956b.

GUIMARÃES, P.S.; PATERNIANI, M.E.A.G.Z.; LÜDERS, R.R.; SOUZA, A.P.; LABORDA, P.R.; OLIVEIRA, K.M.; Correlação da heterose de híbridos de milho com divergência genética entre linhagens. Pesquisa Agropecuária Brasileira, Brasília, v.42, n.6, p.811-16, 2007.

HALLAEUR, A.R. e MIRANDA FILHO, J.B. Quantitative Genetics in Maize Breeding. 2.ed. Ames: Iowa State University Press, 1995. 468 p.

HOISINGTON, D.; KHAIRALLAH, M.; GONZALEZ-DE-LEON, D. Laboratory Protocols: CIMMYT Applied Molecular Genetics Laboratory. 2.ed. México, DF: CIMMYT, 1994. p.2-3.

JACCARD, P. Nouvelles recherches sur la distribution florale. Bulletin Society Vaud Science Natural, v.44, p.223-270, 1908 .

LABORDA, P.R.; OLIVEIRA, K.M.; GARCIA, A.A.F.; PATERNIANI, M.E.A.G.Z.; SOUZA, A.P. Tropical maize germoplasm: what can we say about its genetic diversity in the light of molecular markers? Theoretical and Applied Genetics, Berlin, v.111, p.1288-1299, 2005.

LANZA, L.L.B.;SOUZA JR., C.L.; OTTOBONI, L..M..M.; VIEIRA, M.L.C.; SOUZA, A.P.; Genetic distance of inbred lines and prediction. of maize single-cross performance using RAPD markers. Theoretical and Applied Genetics, Berlin, v.94, n.8, p.1023-1030, 1997.

MANLY, B.F.J. Randomization and Monte Carlo Methods in Biology. London: Chapman \& Hall, 1991. 281p.

MANTEL, N. The detection of disease clustering and a generalized regression approach. Cancer Research, v.27, p.209220, 1967.

MELCHINGER, A.E.; BOPPENMAIER, J.; DHILLON, B.S.; POLLMER, W.G.; HERRMAN, R.G. Genetic diversity for RFLPs in European maize inbreds. II. Relation to performance of hybrids within versus between heterotic groups for forage traits. Theoretical and Applied Genetics, Berlin, v.84, n.5-6, p.672-681, 1992. 
MELCHINGER, A.E.; LEE, M.; LAMKEY, K.R.; HALLAUER, A.R.; WOODMAN, W.L. Genetic diversity for restriction fagment length polymorphisms and heterosis for two diallel ets of maize inbreds. Theoretical and Applied Genetics, Berlin, v.80, n.4, p.488-496, 1990.

MELO, W.M.C.; PINHO, R.G.V.; FERREIRA, D.F. Capacidade combinatória e divergência genética em híbridos comerciais d milho. Ciência Agrotécnica, Lavras, v.25, n.4, p.821-830, 2001.

MILLER, M.P. TFPGA - Tools For Population Genetic Analyses. v.13. Flagstaff: Northern Arizona University, 1997.

MRANDA FILHO, J.B.; GERALDI, I.O. An adapted model for th analysis of partial dialel crosses. Revista Brasileira de Genética, Ribeirão Preto, v.7, n.4, p.677-88, 1984.

MOL, R.H.; LONNQUIST, J.H.; VELEZ FORTUNO, J.; JHNSON, E.C. The relationship of heterosis and divergence in maize. Genetics, New York, v.52, p.139-144, July 1965.

OIVEIRA, K.M., LABORDA, P.R., GARCIA, A.A.F., ZAGATTOPATERNIANI, M.E.A.G.; Souza, A.P. Evaluating genetic rlationships between tropical maize inbred lines by means of AFLP profiling. Hereditas, Harlow, v.140, p.24-33, 2004.

INTO, R.M.C.; GARCIA, A.A.F.; SOUZA JÚNIOR, C.L. de. locação de linhagens de milho derivadas das populações BR105 e BR-106 em grupos heteróticos. Scientia Agricola, Piracicaba, v.58, n.3, p.541-548, 2001.

RAO, R.C. Advanced statistical methods in biometrical research. New York: John Willey and Sons, 1952. 390 p.

EIF, J.C.; MELCHINGER, A.E.; XIA, X.C.; WARBURTON, M.L.; HOISINGTON, D.A.; VASAL, S.K.; SRINIVASAN, G.; BOHN, M.; FRISCH, M. Genetic distance based on simple sequence repeats and heterosis in tropical maize populations. Crop Science, Madison, v.43, p.1275-1282, 2003.

ROHLF, F.J. NTSYSpc - Numerical Taxonomy and Multivariate Analysis System. v. 2.1. New York: Exerter Publications, 1997.

SANTOS, J.B.; VENCOVSKY, R.; RAMALHO, M.A.P. Controle gnético da produção de grãos e de seus componentes primários em feijoeiro. Pesquisa Agropecuária Brasileira, Brasília, v.20, p.1203-1211, 1985.

SCAPIM, C.A.; PACHECO, C.A.P.; TONET, A.; BRACCINI, A.D.L.; PINTO, R.J.B. Análise dialélica e heterose de populações de milho-pipoca. Bragantia, Campinas, v.61, n.3, p.219-230, 2002.

SMOUSE, P.E.; LONG, J.C.; SOKAL, R.R. Multiple regression and correlation extensions of the Mantel test of matrix correspondence. Systematic Zoology, New York, v.35, p.627$632,1986$.

VOS, P.; HOGERS, R.; BLEEKER, M.; REIJANS, M.; VAN DE LEE, T.; HORNES, M.; FRITJERS, A.; POT, J.; PELEMAN, J.; KUIPER, M.; ZABEU, M. AFLP: a new technique for DNA fingerprinting. Nucleic Acids Research, Eynsham, v.23, p.44074414, 1995.
SPRAGUE, G.F. e TATUM, L.A. General vs. specific combining ability in single crosses of corn. Journal of American Society of Agronomy, Madison, v.34, n.10, p.923-932, 1942.

VENCOVSKY, R. Alguns aspectos teóricos e aplicados relativos a cruzamentos dialélicos de variedades. 1970.110p. Tese (LivreDocência) - ESALQ/USP, Piracicaba, 1970. 